\title{
Protocol for the fabrication of enzymatically crosslinked gelatin microchannels for microfluidic cell culture
}

\author{
Amy L Paguirigan \& David J Beebe \\ Department of Biomedical Engineering, University of Wisconsin, Engineering Centers Building, 1150 Engineering Dr., Madison, Wisconsin 53706, USA. \\ Correspondence should be addressed to D.J.B. (djbeebe@wisc.edu). \\ Published online 12 July 2007; doi:10.1038/nprot.2007.256

\begin{abstract}
We have developed a technique for fabricating microfluidic devices from gelatin using a natural crosslinking process. By producing reusable poly(dimethyl siloxane) molds using standard photolithography, gelatin can be molded into microchannel geometries. The gelatin is crosslinked with the naturally occurring enzyme transglutaminase via a straightforward process that can produce devices suitable for cell culture. The protocol takes approximately 1 day from the start of gelatin preparation to cell seeding. Using these devices, the effects of both the extracellular matrix and soluble factors on cellular behavior and differentiation can be studied in
\end{abstract} \\ microenvironments that more closely mimic the in vivo environment.
}

\section{INTRODUCTION}

This protocol describes in additional detail a previously published method for fabricating enzymatically crosslinked gelatin microchannels ${ }^{1}$. Gelatin-based microchannel culture is a cell culture platform that includes a flexible protein substrate and high cell volume density provided by the micromolded channels in the gel substrate. The technique is based on the application of photolithography to form molds of poly(dimethyl siloxane) (PDMS), which then can be used several times to act as a mold for a gelatin solution while it is enzymatically crosslinked. After cells are seeded into these devices, it has been shown that cells can invade the matrix and form three-dimensional (3D) structures ${ }^{1}$. These devices were fabricated to try to mimic the in vivo microenvironment in an in vitro cell culture platform.

The in vivo microenvironment of mammary epithelial cells, much like that of many other tissues in the body, consists of a wide variety of interactions and stimuli. Cell-cell interactions can occur via mechanical contact between either similar cell types (homotypic) or dissimilar cell types (heterotypic). Soluble factors produced and received in these types of interactions along with those received from more distant cells (e.g., stromal-epithelial) can provide an infinitely diverse range of signals and instructions for proliferation, organization, polarization, motility and numerous unknown signals and functions. Also important to cell behavior are cell-matrix interactions, which can provide both structural support and direct signaling and expression. Lastly, mechanisms of mass transport are critical for cell survival, both for providing nutrients and endocrine signals and for removing waste products and signaling molecules produced by the cells of interest. This protocol describes how to make gelatin microchannels for microfluidic cell culture, which provides both a defined matrix (crosslinked collagen) and spatially defined culture areas.

Observations of differentiation, morphology and gene expression due to changes in microenvironment have provided evidence that the microenvironment plays a major role in determining cell fate and behavior ${ }^{2}$. A basic limitation of most in vitro assays of cell behavior or expression is the inability to correlate results to in vivo behavior because of the differences between the in vitro and in vivo microenvironments. This is because traditional macroscale and current microscale in vitro adherent cell culture involves using relatively undefined media over a thin layer of cells grown on rigid, synthetic surfaces (e.g., treated polystyrene). Neither a 3D extracellular matrix (ECM) nor physiological levels of soluble factors are generally present in these types of cultures, resulting in a significantly altered microenvironment from that experienced in vivo.

This limits the ability to accurately understand regulation mechanisms and differentiation pathways occurring in vivo using in vitro assays, thus limiting the development of potential therapeutics for cancer or degenerative diseases.

While microfluidics has shown considerable promise as a tool for studying cell biology, microfluidics' potential to create more in vivo-like in vitro environments has remained largely untapped. One major difference between the macroscale and microfluidic cultures is the importance of diffusion at the microscale as the predominating mass transport mechanism rather than convective transport found in macroscale cultures (Fig. 1 $)^{3-5}$. In the absence of convective mass transport, local concentrations of components added to the media, secreted by the cells or present in surface treatment materials, along with those components remaining from device fabrication, could potentially have more pronounced effects on cellular response than in traditional cultures. The potential artifacts induced by these uncontrolled factors are often ignored or result in simply higher rates of cytotoxicity. In addition, although it has been shown that effects of the ECM are of critical importance to in vivo tissue function ${ }^{6,7}$, the integration of ECM materials into microfluidic systems for tissue engineering and cell biology is just emerging 8,9 . As new fabrication methods are presented that allow more in vivo-like microenvironments to be produced, our abilities to perform cellular analyses will improve the accessibility of in vivo responses of cells and tissues to stimuli that are impossible to study effectively using current in vitro methods.

\section{Discussion of material choices}

Recent work creating natural polymers has employed a purified natural enzyme, microbial transglutaminase (mTG), to crosslink 


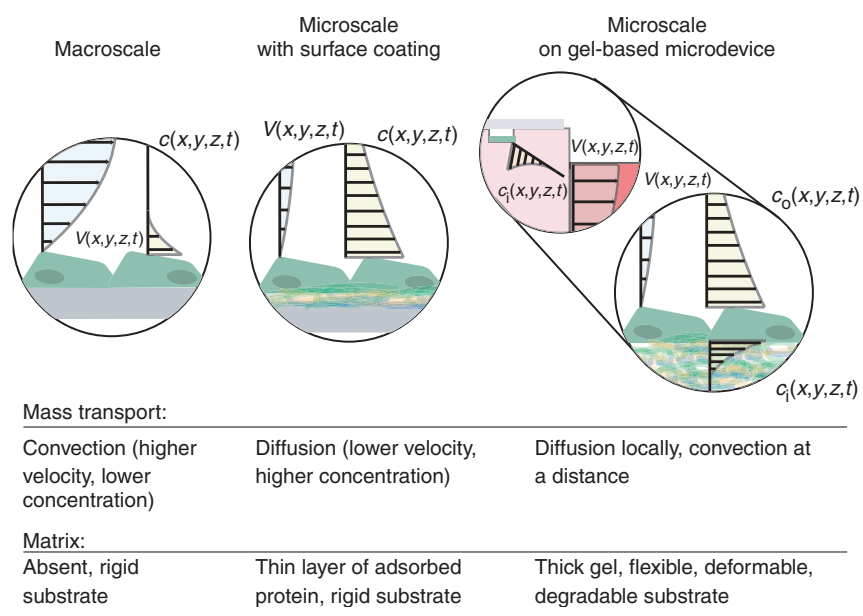

Figure 1 | Current in vitro methods of cell culture and analysis typically have compromised mimicking the cell-matrix interactions and the mechanisms of mass transport owing to limited fabrication techniques. In traditional macroscale cultures, large media volumes are used, resulting in mass transport via convection as the primary mechanism. This results in the local reduction and overall homogenization of any species produced by the cells grown on the bottom surface into the surrounding media. In addition, typically no matrix is provided but only a rigid plastic substrate. New fabrication techniques have allowed microscale culture to be performed, which decreases the convective transport present, allowing diffusion to dominate. This allows concentration gradients of factors near the cells to form via diffusion, closer to how mass transport occurs locally in vivo (although factors are diffusing only through liquid rather than a gel-like matrix). However, these cultures too often do not provide a matrix, but only a rigid substrate. If a matrix is provided (similarly to macroscale cultures), it is simply a thin layer of adsorbed protein on the surface. Using the gel-based microdevice described in this protocol, more in vivo-like transport phenomena can be utilized (diffusion in a gel locally, and convection in a liquid at a distance), and cells can be seeded on a flexible gel matrix that is both deformable and degradable by the cells (figure variables: fluid velocity as a function of space and time, $V(x, y, z, t)$, soluble factor concentration as a function of space and time, $c(x, y, z, t)$, here $c_{\mathrm{i}}(x, y, z, t)$ is the concentration in the gel, whereas $c_{0}(x, y, z, t)$ is the concentration in the surrounding media.

gelatin and other cell-adhesive molecules to create more biocompatible, injectable tissue scaffolding ${ }^{10}$ or adhesives ${ }^{11}$. Preliminary results involving micromolding of gelatin using transglutaminase have also been produced (G.F. Payne, University of Maryland, College Park, Centre for Biosystems Research, personal communication). mTG is currently used in commercial food applications to crosslink proteins found in milk and meat to improve texture or for molding. Also, it has been used to produce biodegradable or edible films, or bioplastics $^{12}$, and has been shown to improve material properties of solid protein materials such as soy proteins for these purposes ${ }^{13}$.

Enzymatically crosslinked gelatin gels have been shown to be biocompatible to both human and bacterial cells, and both have been crosslinked into 3D matrices of gelatin with good cell survival $^{10,14,15}$. Gelatin/mTG biopolymer systems have a distinct advantage over previous synthetic hydrogel systems because of their superior biocompatibility and low costs. For these reasons, we have chosen gelatin (a derivative of collagen) and mTG as the materials for our microdevices. Fabricating microdevices from collagen itself would be prohibitive at current costs. The high cost of purified collagen and the amount of material needed make gelatin the preferred substance over collagen for cell culture.

In vivo, transglutaminase is present in several forms in tissue, blood, ECM and on cell surfaces. All proteins in the transglutaminase family have transamidating activity and catalyze the formation of $N-\varepsilon-(\gamma-$ glutamyl)lysine protein crosslinks (Fig. 2). Members of the transglutaminase family are expressed in many tissues and have many functions in processes such as post-translational modification of proteins, cell adhesion and migration, ECM remodeling and blood clotting at wounds (fibrin polymerization $)^{16-18}$. The transamidating activity of this enzyme is relatively evolutionarily conserved, and mTG has transamidating ability similar to that of the mammalian versions.

For transglutaminase to have crosslinking activity when mixed with a protein solution, the substrate proteins must have glutamine and lysine present. Various proteins found naturally in the ECM or basement membrane of epithelial cells contain varying amounts of these amino acids (Table 1). The higher the percentage composition of each of the amino acids in a protein, the greater the number of potential crosslinking sites, and thus greater the potential to create stiffer gels when supplied with sufficient amounts of transglutaminase, time and environmental conditions.

\section{Additional techniques required}

This protocol relies upon the fabrication of the PDMS molds using standard photolithography techniques that have been the basis for microfluidic culture platforms previously ${ }^{19}$. The basic protocol for making the masters for the PDMS molds is summarized below. It is based on the standard protocol from Microchem Inc. for $250 \mu \mathrm{m}$ feature thickness and SU-8 100. For further information on the preparation of masters for use as PDMS molds, see ref. 19 and http://www.microchem.com/products/pdf/SU8_50-100.pdf.

\section{MATERIALS}

\section{REAGENTS}

- SU-8 100 (Microchem) (mold fabrication)

- Developer (Microchem) (mold fabrication)

- PDMS prepolymer and curing agent (mold fabrication)

- Isopropyl alcohol (mold fabrication)

- Gelatin (Type A from porcine skin, 300 bloom; Sigma) (gelatin fabrication)

- $1 \times$ Dulbecco's phosphate-buffered saline (DPBS) (gelatin fabrication)

- Chloroform (gelatin fabrication)

- mTG (Activa-TI, Ajinomoto Inc., activity of approximately $100 \mathrm{U} \mathrm{g}^{-1}$ of

mTG according to the manufacturer) (gelatin fabrication)

- Deionized water (gelatin fabrication)

- Trypsin $0.05 \%(\mathrm{w} / \mathrm{v})$ with EDTA (cell culture)

EQUIPMENT

- Silicon wafers (mold fabrication)
- UV source (mold fabrication)

- Photomasks, printed at 2,400 dpi or higher (mold fabrication)

- Spinner (mold fabrication)

- Shaker (mold fabrication)

- Hot plates (mold fabrication)

- Petri dishes (glass for developing and plastic for curing PDMS) (mold

fabrication)

- Hot plate (gelatin fabrication)

- Glass beakers (2) (gelatin fabrication)

- Syringe (5 ml) (gelatin fabrication)

- Needle (any gauge) (gelatin fabrication)

- Syringe filter $(0.2 \mu \mathrm{m})$ (gelatin fabrication)

- Centrifuge tube (15 ml) (gelatin fabrication)

- Petri dishes (gelatin fabrication) 
- Thermometer (gelatin fabrication)

- Incubator, set at $37^{\circ} \mathrm{C}$ for gelatin crosslinking (gelatin fabrication)

- Hemacytometer (cell culture)

- Centrifuge tubes (cell culture)

- Centrifuge (cell culture)

- Pipettes/tips (cell culture)

- Cell culture hood (cell culture)

- Incubator (if cell culture is to be performed at a temperature other than

$\left.37^{\circ} \mathrm{C}\right)$ (cell culture)

REAGENT SETUP

Cell culture Appropriate cell culture media for the cell type used

(in this case, cells used were normal murine mammary gland epithelial cells (NMuMGs)).

Dulbecco's modified Eagle's medium.

Fetal bovine serum-10\% v/v.

Insulin (from bovine pancreas; Sigma) $-10 \mathrm{mg} \mathrm{ml}^{-1}$.

\section{PROCEDURE}

\section{Fabrication of the PDMS mold $\bigcirc$ TIMING 6 hrs}

1) Spin-coat silicon wafers with negative photoresist, SU-8 100 (Microchem) using the standard Microchem protocol for $250 \mu \mathrm{m}$ feature depth (Fig. 3, specifically panel a).

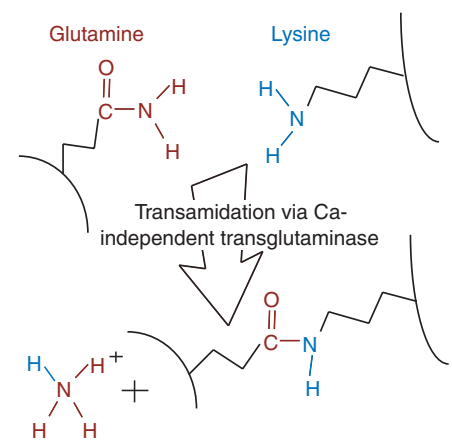

Figure 2 | Glutamine residues in proteins can be covalently linked to lysine residues via a transamidation reaction that results in the production of ammonia. The $\gamma$-carboxamide groups of the glutamine residues act as acyl donors, and the $\varepsilon$-amino groups of lysine residues act as acceptors. The microbial version of transglutaminase is calcium independent (unlike in members of the transglutaminase family found in mammals) and can form both intra- and intermolecular $N$ - $\varepsilon$-( $\gamma$-glutamyl)-lysine crosslinks.

2| Use photomasks (an example is included in Fig. 4) printed at 2,400 dpi or higher to produce the negative of the PDMS mold desired by exposing the wafer to an appropriate dose of UV light ( $365 \mathrm{~nm}$ bandpass with $30 \mathrm{~nm}$ band width on a Novacure) as per Microchem's protocol.

3| Cure and develop master as per Microchem's protocol (Fig. 3b).

4| Mix PDMS prepolymer and curing agent (with 1:10 curing agent to prepolymer weight ratio) well and degas in a vacuum desiccator for 5 min or until all bubbles are removed.

5| Cure the PDMS on the master on a hotplate (at $85^{\circ} \mathrm{C}$ for $90 \mathrm{~min}$ ) (Fig. 3c).

$\triangle$ CRITICAL STEP Make sure the hotplate is placed on an even surface before molding, to ensure that the top of the PDMS mold is even. Otherwise, the bottom of the gelatin channels will not be even, resulting in uneven cell seeding.

6| Allow to cool to room temperature $\left(25^{\circ} \mathrm{C}\right)$ and remove PDMS from the master.

7| Sterilize PDMS (autoclave or spray with ethanol briefly and allow to dry in a biological hood under UV) (Fig. 3d).

- PAUSE POINT Once the molds are made and sterilized, this is a good place to stop overnight. Molds can be stored in a sterile container indefinitely if not opened outside of a biological hood.

\section{Gelatin preparation TIMING Steps 8-11, 45 min; Steps \\ 12-16, $7 \mathrm{hr}$}

8| Make the gelatin/mTG prepolymer mixture using a

$12.5 \mathrm{wt} \%$ gelatin (Type A from porcine skin, 300 bloom,

TABLE 1 | Amino-acid composition of common extracellular matrix and basement membrane proteins.

\begin{tabular}{lcc}
\hline Protein & Glutamine (\%) & Lysine (\%) \\
\hline Collagen, rat, type 1 $^{\mathrm{a}}$ & 3.7 & 4.4 \\
Fibronectin, bovine $^{\mathrm{b}}$ & 5.6 & 3.4 \\
Laminin, murine $^{\mathrm{c}}$ & 5.3 & 4.2 \\
Gelatin-300 bloom $^{\mathrm{d}}$ & 8.4 & 2.6 \\
Gelatin-175 bloom $^{\mathrm{d}}$ & 8.3 & 2.8 \\
\hline
\end{tabular}

average of the content in all types of $\alpha 1$ and $\alpha 2$ chains (from UniProtKB/Swiss-Prot). ${ }^{\text {b From }}$ UniProtKB/Swiss-Prot. 'Average of all types of all chains $(\alpha, \beta, \gamma)$ (from UniProtKB/Swiss-Prot). ${ }^{\mathrm{d} B}$ Bloom refers to the practice of 'grading' gelatin based on its ability to form a stiff gel when mixed with water. The higher the bloom, the higher the stiffness gel it can form when mixed with water. Proteins with lysine and glutamine residues can be crosslinked using the mTG used here. The amount of these amino acids present in the proteins of interest will determine how efficient the crosslinking will be and the maximum stiffness that can be attained. Percentages are from commonly used sources of these proteins (collagen 1 from rat tail, fibronectin from bovine serum and laminin from Engelbreth-Holm-Swarm murine sarcoma basement membrane, Sigma). The values for gelatin were determined by hydrolysis of commercial gelatin isolated from porcine skin. This material would not be a purified, single-collagen type such as that found in commercially available rat tail collagen, but it would contain a variety of ECM components that were not removed during processing.

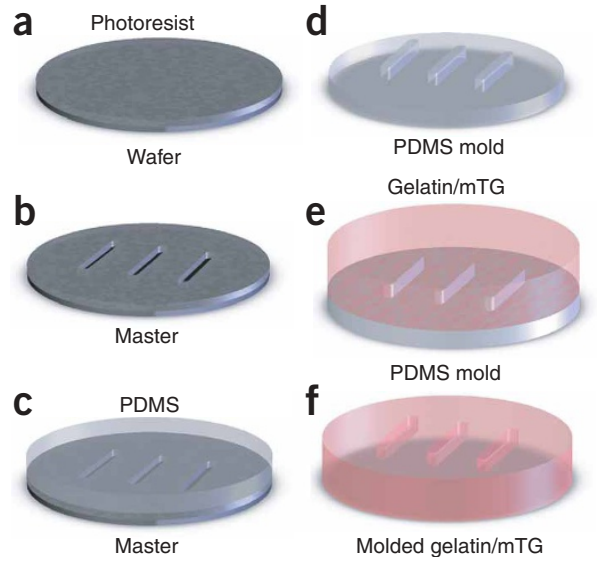

Figure 3 | Schematic of the molding procedure. SU-8 100 photoresist is spin-coated onto silicon wafers (a), and UV exposure through a photomask and developing produces a master (b). PDMS is then cured on this master (c), and used as a mold for gelatin (d). The gelatin and enzyme solution is then poured over the reusable, flexible PDMS mold (e), and the polymerized device is then pulled off the mold $(\mathbf{f})$. 
Sigma) solution in $1 \times$ DPBS (e.g., $1 \mathrm{~g}$ of gelatin powder in $8 \mathrm{ml}$ DPBS).

9| Heat this solution to not higher than $70{ }^{\circ} \mathrm{C}$ to solubilize and fully hydrate the gelatin.

10| Add $0.02 \%$ (vol/vol) chloroform to sterilize the solution (e.g., $2 \mu \mathrm{l}$ of chloroform to $9 \mathrm{ml}$ gelatin/DPBS solution). $\triangle$ CRITICAL STEP Allow gelatin and chloroform mixture to hydrate for $30+$ min to allow the maximal amount of chloroform to evaporate from the solution.

11 Dissolve the mTG in $1 \times$ DPBS using the appropriate mass of mTG to give $10 \mathrm{U}$ mTG per gram of gelatin (e.g., $0.1 \mathrm{~g} \mathrm{mTG}$ in $1 \mathrm{ml}$ DPBS). The final concentrations of the prepolymer mixture used in molding were $10 \mathrm{wt} \%$ gelatin, with $10 \mathrm{U}$ mTG per gram of gelatin. This will produce $10 \mathrm{ml}$ of total solution, which can be poured over molds in three to four $60 \mathrm{~mm}$ Petri dishes to make gelatin gels approximately $2 \mathrm{~mm}$ thick.

12| Pour the resulting mixture over the PDMS molds in Petri dishes (Fig. 3e).

$\triangle$ CRITICAL STEP This must be done quickly, and the two solutions must be mixed thoroughly before gelation begins to prevent any further mixing. Initial gelation will occur within $5 \mathrm{~min}$, which means that the solutions must be mixed well, poured and set on a level surface before this time.

13| Incubate at $37^{\circ} \mathrm{C}$ for $5 \mathrm{~h}$.

$\triangle$ CRITICAL STEP Level incubator shelves before molding to ensure that the top of the gelatin is even. If they are not leveled, then the channel bottoms will not be even, resulting in uneven cell seeding. Note: Initial gelation should occur within 5 min, but the a

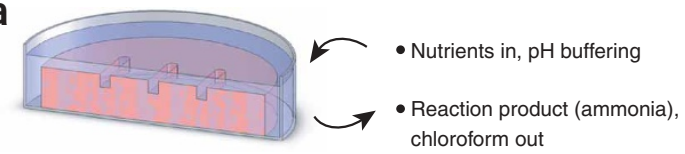

b

Soak in cell culture media

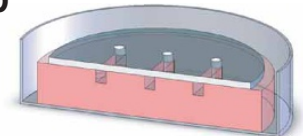

Add thin PDMS top

C

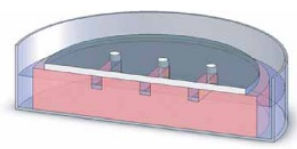

Add reserve media

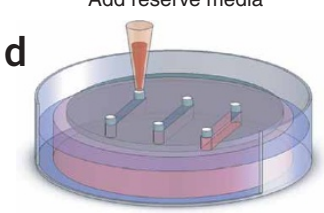

Add cell suspension

e

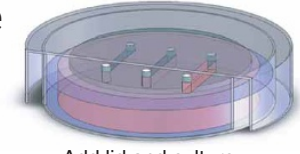

Add lid and culture
- Reduces evaporation

- Aids in wicking/pumping of media and cell suspension into channels

- Allows diffusional transport of nutrients and waste products to the sink

- $\mathrm{pH}$ buffer, counteracts/reduces effects of evaporation

- Wick in media drop

- Add cell suspension using passive pumping mixture will need to be incubated longer to obtain higher degrees of crosslinking and higher stiffness to facilitate removal from the molds. If sufficient stiffness is not attained, the gels will tear when removed from the molds.

14| After incubation, the gelatin/PDMS should be cooled to $4{ }^{\circ} \mathrm{C}$.

15| Carefully remove the crosslinked gelatin from the PDMS mold (Fig. 3f).

16| Heat-treat the crosslinked gelatin gels in $1 \times$ DPBS for $30 \mathrm{~min}$ at $65^{\circ} \mathrm{C}$ to heat-inactivate the remaining enzyme and to dialyze any remaining chloroform from the gels, along with any reaction product (ammonia) still present.

17| Wash the PDMS molds using water and a dilute detergent such as $0.1 \mathrm{wt} \%$ Alconox. Re-sterilize them via autoclaving or ethanol/UV exposure and store in a sterile container. When washed of the residual protein, molds can be reused unless they are damaged by handling. Normal molding steps usually will not affect the PDMS significantly in our experience.

\section{Pretreatment of crosslinked gelatin gels $\bigcirc$ TIMING 2 hrs-overnight}

18| Place the crosslinked gelatin gel microchannels with the molded side up into a bath of the cell culture media. Soak until the gel has equilibrated with the media (if the medium has phenol red in it, wait until the gel is completely pink) (Fig. 5a).

tions for specific steps in the pretreatment crosslinked gelatin gel protocol. (a) Soak the gels in the culture media to remove reaction products. (b) Add PDMS membrane to enclose channels to enable wicking of media into channels and reducing evaporation. (c) Add reserve media around the base of the gelatin to provide a nutrient source and waste sink for culture. (d) Add cell suspension using passive pumping. (e) Close dish and place in incubator.

$$
\begin{aligned}
& \text { incubator } \\
& \text { Check reserve media for color } \\
& \text { change, replace to continue to } \\
& \text { buffer culture }
\end{aligned}
$$
but it is not advisable to leave gelatin for a longer period in the media, as any accidental contamination (during fabrication or afterwards) will degrade the gel. 
a

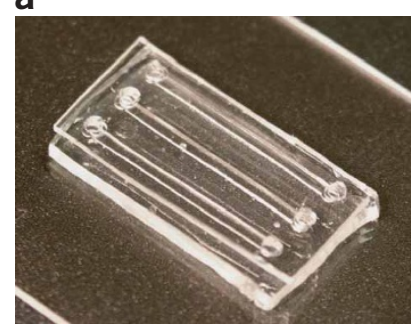

b

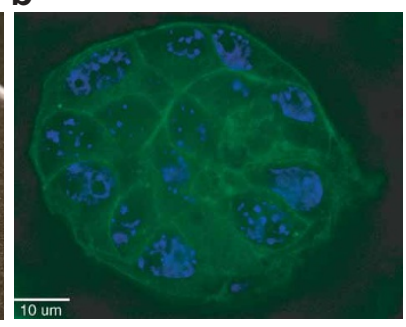

C

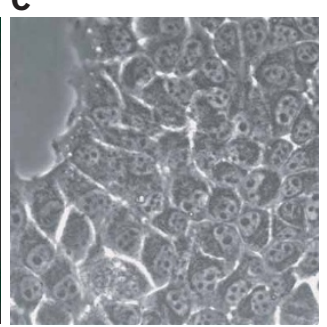

f

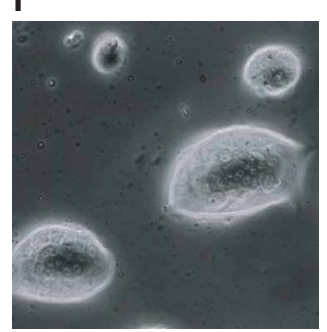

d

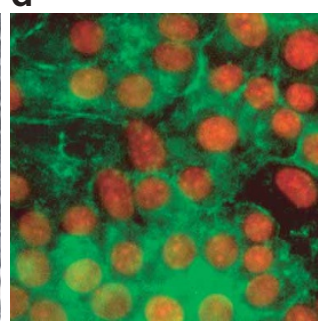

g

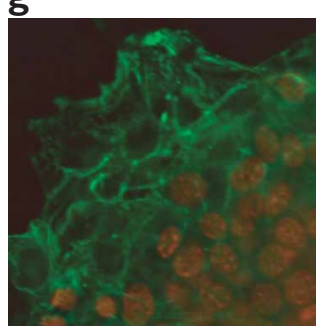

e

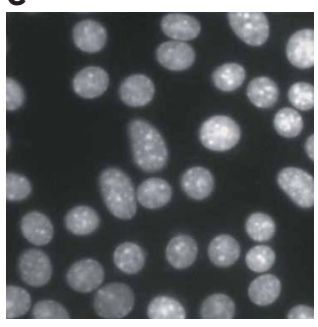

h

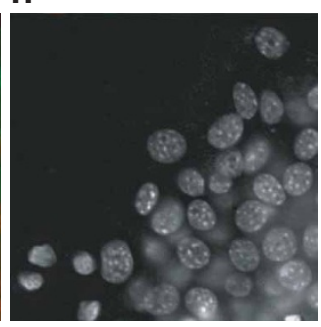

Figure 6 | Overall device design and representative images of NMuMG epithelial cells grown on traditional TC plastic and in gelatin microchannels. Example of a set of channels in gelatin with a PDMS membrane with ports cored (a), and a 3D deconvoluted image of NmuMGs grown in gelatin channels at 3 days post-seeding, stained for actin (green) and nuclei (blue) (b). Cells grown on traditional TC-treated plastic form a monolayer (shown at $\times 20$ magnification, in phase (c), actin (green) and nuclei (red) (d) and just the nuclei (red channel) (e)). All the nuclei in $\mathbf{e}$ are in the same focal plane, and the actin cytoskeleton in $\mathbf{d}$ shows more diffuse staining, with few projections. However, cells grown in gelatin microchannels show invasion into the matrix (shown at $\times 20$, in phase (f), actin (green) and nuclei (red) (g) and just the nuclei (red channel) (h)). The nuclei in $\mathbf{h}$ are overlapping and some are in focus, whereas others are not, showing that they are in different focal planes and the cells have formed many processes as they expand into the gel (actin in $\mathbf{g}$ ). Images reproduced from Paguirigan and Beebe (2006) with the permission of the Royal Society of Chemistry.

19| Drain off excess cell culture media.

20| Add a thin (approximately $500 \mu \mathrm{m}$ ) membrane of PDMS with ports cored at the appropriate locations to the top to form a complete fluidic channel (Fig. 5b). Note: The PDMS membranes will adhere to the surface of the gelatin loosely, but only if dry. Once they are wet, they will slide when put on the surface.

21| Add reserve media to fill Petri dish to a level below the top of the gelatin channels (Fig. 5c).

$\triangle$ CRITICAL STEP Make sure medium is not added in excess so that when the dish is handled (e.g., taken to the incubator or microscope) it does not splash on top of the gelatin/PDMS.

22| Add media to the channels. The media should wick (capillary action) into the channels after simply placing a drop of media at one of the ports.

\section{Cell seeding $\odot$ TIMING $\mathbf{3 0}$ min}

23| Trypsinize adherent cells to remove them from flask culture by adding trypsin solution and incubating at $37^{\circ} \mathrm{C}$ for $1-2$ min.

24| Add media to inactivate the trypsin and dilute it.

25| Take out $5 \mu \mathrm{l}$ and use a hemacytometer to count cells.

26| Centrifuge to pellet the cells (for NMuMGs, $200 \mathrm{~g}$ for $7 \mathrm{~min}$ is sufficient to pellet $5 \mathrm{ml}$ of cells at $1 \mathrm{million}^{-1}$ ).

27| Resuspend at the desired concentration for seeding.

$\triangle$ CRITICAL STEP Filling the channels (without bubble formation or excess shear on the cells) is best done by first adding cell culture media, then cell suspension; so when resuspending cells, include this dilution factor when calculating the desired stock concentration (Fig. 5d).

28| Add cell suspension. Note that the PDMS membrane allows passive pumping to be used to pump in the cell suspension drop (add a small drop to one port, and allow it to pump via surface tension-driven flow to the opposite port, thus filling the channel).

29| Close the lid of the Petri dish (Fig. 5e).

30| Place the dish into a humidified incubator with the appropriate settings for the cell type used (typically for mammalian cells this will be $37^{\circ} \mathrm{C}$ and $5 \% \mathrm{CO}_{2}$ ). 
TIMING

Fabrication of PDMS mold: approximately $6 \mathrm{~h}$

Gelatin preparation:

Steps 8-11: $45 \mathrm{~min}$

Steps 12-16: $7 \mathrm{~h}$

Pretreatment of gelatin gels: from $2 \mathrm{~h}$ to overnight (if desired)

Cell seeding: $30 \mathrm{~min}$

\section{? TROUBLESHOOTING}

Troubleshooting advice can be found in Table 2 .

TABLE 2 | Troubleshooting table.

\begin{tabular}{lll}
\hline Problem & Possible reason & Solution \\
\hline $\begin{array}{ll}\text { Gel has pits of dissolved gelatin across } \\
\text { its top }\end{array}$ & $\begin{array}{l}\text { Contamination, bacteria are degrading the } \\
\text { gel locally }\end{array}$ & $\begin{array}{l}\text { Add more chloroform, up to } 0.08 \% \text { (vol/vol), } \\
\text { to the gelatin solution, and leave for a further } \\
\end{array}$ \\
& & 30 min before pouring \\
Buy a new lot of gelatin, as the previous lot \\
might have very high levels of contamination \\
\end{tabular}

Gel is uneven, with softer and harder parts present

Gelatin solution and enzyme solution were not completely mixed before gelation

Enzyme solution's activity is too low, owing to age, oxygen exposure, high heat, storage in dissolved form
Mix two solutions via pipetting with a largeorifice serological pipette before pouring-DO NOT SHAKE, or the bubbles that are formed will remain after crosslinking

Store the enzyme in a sealed container, at $-20{ }^{\circ} \mathrm{C}$ or lower if long-term storage is intended

Acquire a new enzyme lot

Mix the enzyme and DPBS immediately before use, do not use solutions prepared earlier

\section{ANTICIPATED RESULTS}

An example of how the devices look after the PDMS membrane is applied to the top of the gelatin microchannels is shown in Figure 6a. Once the cells have been seeded into the gelatin channels, they should be allowed to adhere for up to $24 \mathrm{~h}$ before any media changes or treatment. As the cells are allowed to proliferate and invade the gelatin, they will form more 3D structures (Fig. $\mathbf{6 b}$ ) than on traditional TC plastic shown after 3 days of culture (Fig. $\mathbf{6 c}-\mathbf{h}$ ). Using a z-stage on a microscope, one can image through these structures, and the use of deconvolution software (such as Slidebook as used in Fig. 6b) can significantly improve the quality of the images taken. Depending on the cell type, structures will form at different rates, but for NMuMGs, after 3 days, the cells in Figure $6 \mathbf{b}$ were approximately $50 \mu \mathrm{m}$ wide and $60-70 \mu \mathrm{m}$ deep.

COMPETING INTERESTS STATEMENT The authors declare no competing financial interests.

Published online at http://www.natureprotocols.com Reprints and permissions information is available online at http://npg.nature.com/ reprintsandpermissions

1. Paguirigan, A. \& Beebe, D.J. Gelatin based microfluidic devices for cell culture. Lab on a Chip 6, 407-413 (2006).

2. Wozniak, M.A. et al. ROCK-generated contractility regulates breast epithelial cell differentiation in response to the physical properties of a three-dimensional collagen matrix. J. Cell Biol. 163, 583-595 (2003).

3. Atencia, J. \& Beebe, D. Controlled microfluidic interfaces. Nature $437,648-655$ (2005).
4. Walker, G., Mensing, G. \& Beebe, D. Physics and applications of microfluidics in biology. Annu. Rev. Biomed. Eng. 4, 261-286 (2002).

5. Walker, G., Zeringue, H. \& Beebe, D. Microenvironment design considerations for cellular scale studies. Lab on a Chip 4, 91-97 (2004).

6. Condon, M.S. The role of the stromal microenvironment in prostate cancer. Semin. Cancer Biol. 15, 132 (2005).

7. Danen, E.H.J. \& Sonnenberg, A. Integrins in regulation of tissue development and function. J. Pathol. 200, 471-480 (2003).

8. Dusseiller, M.R. An inverted microcontact printing method on topographically structured polystyrene chips for arrayed micro-3-D culturing of single cells. Biomaterials 26, 5917-5925 (2005).

9. Tan, W. \& Desai, T.A. Microfluidic patterning of cellular biopolymer matrices for biomimetic 3-D structures. Biomed. Microdev. 5, 235-244 (2003). 
PROTOCOL

10. Ito, A. et al. Transglutaminase-mediated gelatin matrices incorporating cell adhesion factors as a biomaterial for tissue engineering. J. Biosci. Bioeng. 95, 196-199 (2003).

11. McDermott, M.K. et al. Mechanical properties of biomimetic tissue adhesive based on the microbial transglutaminase-catalyzed crosslinking of gelatin. Biomacromolecules 5, 1270-1279 (2004).

12. Lim, L., Mine, Y. \& Tung, M. Barrier and tensile properties of transglutaminase cross-linked gelatin films as affected by relative humidity, temperature and glycerol content. J. Food Sci. 64, 616-622 (1999).

13. Mizuno, A., Mitsuiki, M. \& Motoki, M. Effect of transglutaminase treatment on the glass transition of soy protein. J. Agric. Food Chem. 48, 3286-3291 (2000).

14. Chen, R.N., Ho, H.O. \& Sheu, M.T. Characterization of collagen matrices crosslinked using microbial transglutaminase. Biomaterials 26, 4229-4235 (2005).
15. Chen, T.H. et al. Enzymatic methods for in situ cell entrapment and cell release. Biomacromolecules 4, 1558-1563 (2003).

16. Aeschlimann, D. \& Thomazy, V. Protein crosslinking in assembly and remodelling of extracellular matrices: the role of transglutaminases. Connect. Tissue Res. 41, 1-27 (2000).

17. Belkin, A.M. et al. Matrix-dependent proteolysis of surface transglutaminase by membrane-type metalloproteinase regulates cancer cell adhesion and locomotion. J. Biol. Chem. 276, 18415-18422 (2001).

18. Priglinger, S.G. et al. TGF-beta 2-induced cell surface tissue transglutaminase increases adhesion and migration of RPE cells on fibronectin through the gelatin-binding domain. Invest. Ophthalmol. Vis. Sci. 45, 955-963 (2004).

19. Tourovskaia, A., FigueroaMasot, X. \& Folch, A. Long-term microfluidic cultures of myotube microarrays for high-throughput focal stimulation. Nat. Protoc. 1, 1092-1104 (2006). 